\title{
Cerebral amyloidosis angiopathy related inflammation as a cause of erratic driving in elderly
}

\author{
Stella Pak*, Damian Valencia and Adam Fershko \\ Department of Medicine, Kettering Medical Center, Kettering, Ohio, USA
}

Cerebral amyloid angiopathy is a well-known clinicopathology prevalent in the elderly population. This disease is characterized by $\beta$-amyloid deposits in the vessels supplying blood to the cerebral cortex and the leptomeninges [1]. A rare complication of this disease is cerebral amyloid angiopathy-related inflammation (CAA-ri), which is a reversible encephalopathy caused by inflammation and edema [2]. Common clinical presentations of this complication are headaches, focal neurological deficit, and seizure [1]. Herein, we present a case of a patient who presented with an abrupt onset of driving impairment due to CAA-ri.

A 77 years old female presented with several incidents over 10 day period of erratic driving and slurred speech. While driving, she tried to turn left onto oncoming traffic multiple times, drove into traffic cones, and cut off other drivers. During the week before admission she was observed at times to be moving very slowly and deliberately, although patient was not aware of feeling differently. She specifically denied feeling confused, weak, numb, or off balance. She did, however, report recent worsening of headache associated with visual symptoms. She has been experiencing these episodes of headache over approximately 4 years. The visual symptoms were binocular, off-center, and as "a scintillating pinwheel”, typically lasting for 3-4 minutes. About $40 \%$ of the time the visual symptoms were immediately followed by brief right-sided headache, which she describes as an outward pressure and associated by slight nausea. Her medical history consists of hyperlipidemia, hypertension, and hypothyroidism.

At the time of admission, computerized tomography (CT) of her brain revealed a shift of midline structures by $6.4 \mathrm{~mm}$ to the left with mass effect upon the right lateral ventricles (Figure 1). The abnormal finding from computerized tomography scan prompted further investigation with magnetic resonance imaging (MRI) and angiography. MRI showed significant vasogenic edema on the right side and abnormal white matter signal with innumerable petechial hemorrhages (Figure 2). Cerebral angiogram showed displacement of the large intracranial vessels toward the left side.

Electroencephalogram the day after admission showed focal disturbance of cerebral activity involving the right hemisphere. She was started on Dexamethasone $(2 \mathrm{mg} \mathrm{Q} 8 \mathrm{H})$ and started to show improvement in mental status and headaches. CT of chest, abdomen, and pelvis did not show any abnormal findings, except pre-existing sigmoid diverticulosis. She was started on levetiracetam (500 mg BID). On third day of admission, brain biopsy was taken from her right frontal lobe via craniotomy. The biopsy did not show any evidence for inflammatory or malignant process. However, a Congo-red stain showed patchy weak apple-green birefringence in the congophllic areas of the vasculature. CSF analysis result was negative. On fifth day of admission, she was discharged on dexamethasone and levetiracetam for the next 60 day.

Eight days after her discharge, follow-up examination revealed residual symptoms including headaches and mild confusion. She could not pass driving evaluation due to cognitive impairment at that time. Three weeks following the discharge, she was in normal mental status with no focal neurological deficit. MRI also showed significant decrease in severe white matter signal abnormality in right hemisphere. Two months after the date of discharge, dexamethasone was discontinued, but levetiracetam therapy was continued for seizure prophylaxis.

On follow-up examination 3.5 months after discharge showed new-onset gait instability and tremor and orthostatic hypotension. Gait instability and tremor spontaneously resolved over a few weeks, but orthostatic hypotension persisted even after discontinuing her antihypertensive medications. She also got her driving privileges back after completing a driving evaluation.

This patient showed abrupt onset of erratic driving heralded by increased frequency of headache associated with visual symptoms, most probably due to CAA-ri. Her neuroimaging, brain biopsy result, and remarkable improvement on steroid treatment supported this diagnosis.

This case highlights that CAA-ri can manifest as acute driving impairment, which can lead to dangerous motor vehicle accidents.
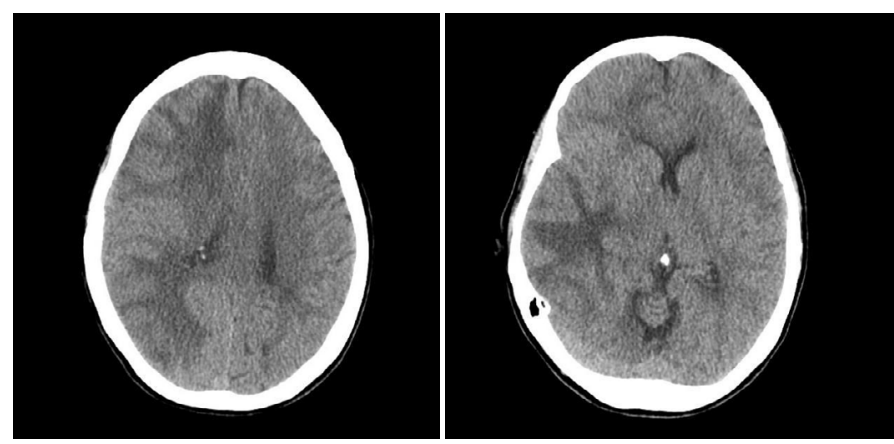

Figure 1. Computerized tomography of brain demonstrating a shift of midline structures by $6.4 \mathrm{~mm}$ to the left, leading to mass effect upon the right lateral ventricles.

Correspondence to: Stella Pak, MD, Department of Medicine, Kettering Medical Center, Kettering, Ohio, USA, Tel. (937) 395-8997; Fax: (937) 395-8399; E-mail: Stella.Pak@ketteringhealth.org

Received: October 20, 2017; Accepted: October 31, 2017; Published: November 03, 2017 

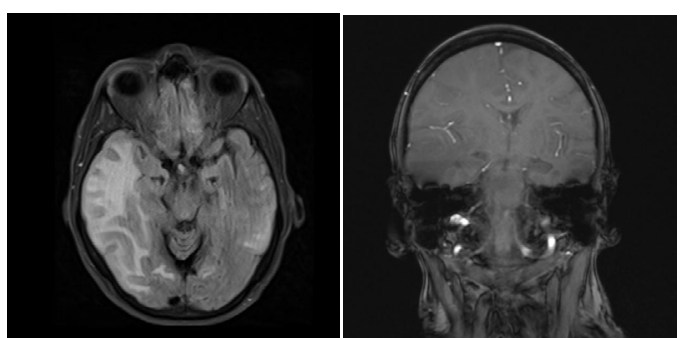

Figure 2. Magnetic resonance imaging of brain significant vasogenic edema on the right side and abnormal white matter signal with innumerable petechial hemorrhages (LeftHorizontal View, Right-Coronal View).
Therefore, it would be crucial to consider the possibility of CAA-ri in elderly patients presenting with erratic reckless driving history. Since this condition can be completely or partially reversed, early diagnosis would greatly benefit the patients with CAA-ri.

\section{References}

1. Chu S, Xu F, Su Y, Chen H, Cheng X (2016) Cerebral amyloid angiopathy (CAA)related inflammation: Comparison of inflammatory CAA and amyloid-beta-related angiitis. J Alzheimers Dis 51: 525-532. [Crossref]

2. Liang JW, Zhang W, Sarlin J, Boniece I (2015) Case of cerebral amyloid angiopathyrelated inflammation - Is the absence of cerebral microbleeds a good prognostic sign? $J$ Stroke Cerebrovasc Dis 24: e319-322. [Crossref]

Copyright: $(02017$ Pak S. This is an open-access article distributed under the terms of the Creative Commons Attribution License, which permits unrestricted use, distribution, and reproduction in any medium, provided the original author and source are credited. 